\title{
ADOÇÃO DO PLANEJAMENTO ESTRATÉGICO SITUACIONAL NA FARMACOVIGILÂNCIA E SEGURANÇA DO PACIENTE DE UM PROJETO ENSINO-SERVIÇO
}

Situational strategic planning adoption in pharmacovigilance and patient safety education a-service project Adopción del planeamiento estratégico situacional en la Farmacovigilancia y

seguridad del paciente de un proyecto enseñanza-servicio

Gisele da Silveira Lemos ${ }^{1,2 *}$, Priscila Ribeiro de Castro ${ }^{2}$, Milena Soares dos Santos ${ }^{3}$

${ }^{1}$ Universidade Estadual do Sudoeste da Bahia (UESB)

2 Universidade Federal da Bahia (UFBA)

${ }^{3}$ Instituto Multidisciplinar em Saúde, Campus Anísio Teixeira, Universidade Federal da Bahia (UFBA)

*E-mail: gisalemos@ig.com.br

\section{RESUMO}

Introdução: Os eventos adversos ocorridos na assistência à saúde são considerados na atualidade como um importante problema de saúde pública, devido principalmente ao número de óbitos, às incapacidades provocadas e aos custos gerados aos sistemas de saúde. A prevenção desse tipo de EA passa por uma abordagem sistêmica, que visa identificar os pontos vulneráveis do processo assistencial e continua como um desafio para os profissionais envolvidos nos serviços de saúde.

Objetivo: Este trabalho teve como objetivo utilizar uma ferramenta de gestão, proposta por Carlos Matus, para a implantação e implementação de um projeto de extensão de Farmacovigilância na Segurança do Paciente em um hospital geral.

Métodos: Para a execução deste trabalho foram realizados os quatro momentos do Planejamento Estratégico Situacional (PES): explicativo, normativo, estratégico e tático-operacional pela equipe do projeto juntamente com os atores do serviço.

Resultados: Observou-se que a ferramenta do PES ofereceu subsídios para o crescimento profissional dos participantes e possibilitou a intervenção de maneira estratégica em problemas do cotidiano dos serviços do hospital.

Conclusão: Dessa forma, o PES pode ser utilizado como uma estratégia de trabalho na graduação de cursos da área de saúde e na gestão de serviços de saúde.

Palavras-chave: Planejamento Estratégico. Farmacovigilância. Hospitais de Ensino. Segurança do Paciente.

\section{ABSTRACT}

Introduction: Adverse events (AEs) occurred in health care are considered today as an important public health problem, mainly due to the number of deaths, caused the disability and costs caused to health systems. The prevention of this kind of AE goes through a systemic approach which aims to identify the vulnerable points of the care process and remains a challenge for the professionals involved in health services.

Objective: This work aimed to use a management tool, proposed by Carlos Matus, for the deployment and implementation of a Pharmacovigilance extension project on the Patient Safety in a general hospital.

Methods: For this assignment, it was performed the four times of the Situational strategic planning (SSP): explanatory, normative, strategic and tactical-operational by the project team together with the stakeholders of the service.

Results: It was observed that the SSP tool offered subsidies for the growth professional of participants and enabled the strategically intervention in hospital services everyday problems.

Conclusion: Thus, the SSP can be used as a working strategy in undergraduate health courses and health services management.

Keywords: Strategic Planning. Pharmacovigilance. Teaching hospitals. Patient Safety. 


\section{RESUMEN}

Introducción: Los acontecimientos adversos que ocurren en el cuidado a la salud,en la actualidad, son considerados un importante problema de salud pública importante, debido, principalmente, al número de muertes, a las discapacidades causadas y a otros gastos causados a los sistemas de salud. La prevención de ese tipo de acontecimientos adversos pasa por un enfoque sistémico, que tiene como objetivo identificar los puntos vulnerables del proceso de atención a la salud y, sigue siendo un reto para los profesionales involucrados en los servicios de salud.

Objetivo: Este trabajo tuvo el objetivo de utilizar una herramienta de gestión, propuesta por Carlos Matus, para despliéguela implantación y ejecución de un proyecto de extensión de farmacovigilancia en la Seguridad del Paciente en un hospital general.

Métodos: Para la ejecución de este trabajo, el equipo del proyecto en conjunto con los actores del servicio realizó cuatro momentos de la Planificación Estratégica Situacional se realizaron (PES): explicativo, normativo, estratégico y táctico-operativo por el equipo del proyecto conjuntamente con los actores del servicio.

Resultado: Se observó que la herramienta del PES ofreció subsidios para el crecimiento profesional de los participantes del proyecto y permitió una intervención estratégica en problemas cotidianos de los servicios del hospital.

Conclusion: Por lo tanto, el PSE se puede ser utilizado como una estrategia de trabajo en cursos de grado de salud y en la gestión de los servicios de salud.

Palabras clave: Planificación Estratégica. Farmacovigilancia. Hospitales de enseñanza. Seguridad del Paciente.

\section{INTRODUÇÃO}

Novas tecnologias na assistência à saúde evidenciam melhoras na prevenção, reabilitação e promoção da saúde, com aumento da expectativa de vida no mundo. No entanto, essas inovações tornam a assistência mais onerosa, complexa e permeada por riscos, ou seja, a probabilidade de ocorrência de um evento ou circunstância que poderia ter resultado, ou resultou, em dano desnecessário à saúde ${ }^{(1,2)}$

A utilização segura e correta de medicamentos é um processo complexo que integra profissionais de várias áreas da saúde exigindo conhecimento sobre os fármacos, uma completa informação sobre o paciente e uma série de decisões interrelacionadas ${ }^{(3)}$.

As consequências das possíveis falhas nos sistemas de saúde trazem impactos negativos tanto para os pacientes e suas famílias, quanto para as organizações e para a sociedade. Estudos apontam que a ocorrência de eventos adversos no processo de atendimento aos pacientes hospitalizados acarreta complicações na evolução de sua recuperação, aumento de taxas de infecções e do tempo médio de internação(4). Essas situações recebem diferentes nomes, como erros médicos, eventos adversos relacionados à internação, agravos à saúde, iatrogenia, erros de medicação e outros. Embora a Organização Mundial de Saúde (OMS) tenha proposto uma padronização dos conceitos-chave para harmonizar a classificação internacional para a segurança do paciente, ainda se observa uma pluralidade de conceitos e definições ${ }^{(5)}$.

A estimativa de que, aproximadamente, uma em cada 10 admissões hospitalares resulta na ocorrência de pelo menos um evento adverso é alarmante, ainda mais se considerarmos que metade desses incidentes poderiam ter sido evitados, segundo estudos conduzidos em hospitais americanos ${ }^{(6)}$. No Brasil, o estudo realizado em três hospitais de ensino evidenciou que a incidência de pacientes com eventos adversos foi de 7,6\%, dos quais 66,7\% foram considerados evitáveis ${ }^{(7)}$.

Dos eventos adversos a medicamentos, os erros de medicação são causa importante de comprometimento da qualidade da atenção ao paciente hospitalizado e, por isso, devem ser identificados e caracterizados. Os erros de medicação ocorridos na 
assistência à saúde são considerados, na atualidade, como um importante problema de saúde pública, devido principalmente às mortes, às incapacidades provocadas e aos custos gerados aos sistemas de saúde. A negação e a subnotificação desses erros na área da saúde prejudicam os estudos na área de segurança do paciente, pois na maioria das vezes não se consegue uma análise real do que está acontecendo(8).

Como alternativa a essa problemática e com vistas ao fortalecimento da Assistência a Saúde de usuários que utilizam os serviços oferecidos pelos sistemas de saúde, a Organização Mundial de Saúde lançou em 2004 o programa Aliança Mundial para a segurança do paciente e em 2013 no Brasil foi publicada a Portaria no 529 de 01 de abril do Ministério da Saúde, que instituiu o Programa Nacional de Segurança do Paciente ${ }^{(9)}$.

Diante deste cenário, um grupo de extensão na Universidade Estadual do Sudoeste da Bahia (UESB), foi criado com objetivo de realizar atividades de farmacovigilância, promover o uso racional de medicamentos e implantar ações para segurança do paciente envolvidas na prescrição, dispensação, uso e administração de medicamentos.

A criação do Programa Nacional de Segurança do Paciente tem como objetivos prevenir e reduzir a incidência de eventos adversos relacionados à assistência nos serviços de saúde. A obrigatoriedade da sua implantação e implementação é estabelecida através da Portaria GM/MS n 3410 de 2013 de contratualização de hospitais no âmbito do Sistema Único de Saúde (SUS), que traz como responsabilidade do hospital a implantação dos protocolos de segurança do paciente ${ }^{(10)}$.

A implantação de serviços para a notificação de erros relacionados ao uso de medicamentos nos hospitais (erros de prescrição, dispensação e administração) geralmente não são bem aceitos por parte dos profissionais que associam as falhas/ erros à vergonha, à perda de prestígio e ao receio de punições. Desse modo, o ambiente nas instituições de saúde é pouco propício para a notificação desses eventos e para a discussão franca sobre o assunto(6).

Como alternativa a essa discussão, o uso do Planejamento Estratégico Situacional (PES) tem grande potencial para processar problemas complexos e pode ser empregado como uma ferramenta auxiliar para a implementação de projetos de ensino serviço(11).

Esse tipo de planejamento se caracteriza por levar em consideração múltiplas dimensões para avaliação dos problemas (política, econômica, social) e análise estratégica dos diferentes atores envolvidos em uma situação de partilha de poder, quadro comum em serviços gerenciados pelo governo ${ }^{(12)}$. Suas características principais envolvem o processo participativo e interativo entre todos os atores envolvidos no serviço, a negociação e pactuação de ações e responsabilidades com base na análise da situação rea|(13).

O presente estudo teve como objetivo descrever o desenvolvimento do plano operacional do PES no projeto de extensão de Farmacovigilância na Segurança do Paciente que atua em um hospital geral da Bahia.

\section{METODOLOGIA}

Este trabalho foi conduzido a partir do exercício de construção de um plano operativo, baseado no Planejamento Estratégico Situacional, para intervenções no projeto de extensão universitária intitulado: Farmacovigilância na segurança do paciente, da Universidade Estadual do Sudoeste da Bahia pela realização do trabalho de conclusão de curso da Especialização em Gestão da Assistência Farmacêutica do Ministério da Saúde e Universidade Federal de Santa Catarina, turma de 2014. Trata-se de um relato de experiência com abordagem metodológica de pesquisa avaliativa em saúde, de caráter observacional. A metodologia do PES é característica de pesquisas voltadas para a avaliação de serviços e processos de saúde por conta da complexidade do fenômeno social investigado(14).

Para a revisão de literatura foi realizada uma busca nas bases de dados SciELO e LILACS através do portal da Biblioteca Virtual em Saúde, utilizando o descritor em saúde planejamento estratégico situacional, no período compreendido entre abril a junho de 2015. 
O serviço de saúde empregado como proposta de intervenção utilizando o PES foi um projeto de extensão universitária de Farmacovigilância e segurança do paciente que atua em um hospital público, vinculado à rede do SUS pela Secretaria Estadual de Saúde da Bahia. Esse hospital atende uma população de cerca de 600 mil habitantes e presta atendimento em 180 leitos ativos nas especialidades de Clínica Médica, Clínica Cirúrgica, Pediatria, Neurologia e Terapia Intensiva. O referido hospital também realiza ensino e pesquisa, recebendo alunos para aulas práticas e estágio dos cursos de graduação em Medicina, Enfermagem, Farmácia e Fisioterapia da Universidade Estadual do Sudoeste da Bahia, além de alunos de enfermagem de mais duas faculdades particulares da cidade.

O intervalo de execução do PES no projeto de extensão foi de agosto de 2014 a fevereiro de 2015, com desenvolvimento de três dos quatro momentos que constituem o plano operativo do Planejamento Estratégico Situacional: momento explicativo, momento normativo, momento estratégico e o momento tático operacional não foi incluído nesta primeira etapa do trabalho de ações voltadas para a implementação do projeto de extensão Farmacovigilância na segurança do paciente, utilizando o plano operativo por meio do planejamento estratégico situacional proposto por Carlos Matus ${ }^{(12)}$ (1996). O momento tático operacional não foi incluído nesta etapa, pois é um processo que requer um tempo longo e para o qual se fazia necessário a disponibilidade de recursos financeiros e humanos ainda não disponíveis.

Os dados foram coletados por meio de uma oficina para identificação, priorização e explicação dos problemas, na qual foi construída a espinha de peixe do PES (problema, descritores, causas, consequências, causa convergente, consequência convergente e imagem- objetivo). Os objetivos, ações e análises de viabilidade e factibilidade do plano operativo foram discutidos em reuniões distintas com os atores.

Todos os atores envolvidos com a implantação e implementação do projeto de extensão no Hospital foram convidados, totalizando quatorze. Dentre eles estavam: coordenador da Farmácia, coordenadora do Núcleo de Segurança do Paciente, coordenadora de Enfermagem, médico da Secretária Estadual de Saúde, alunos de graduação em Farmácia e farmacêutico participantes do projeto.

O projeto de extensão foi submetido à Pró-Reitoria de extensão da Universidade Estadual do Sudoeste da Bahia para financiamento, e foi aprovado com ônus sua realização no período de abril de 2014 a dezembro de 2015.

O projeto de extensão foi submetido no formato de pesquisa para o Comitê de Ética em Pesquisa da UESB em consonância com a Resolução do Conselho Nacional de Saúde (CNS) n 466/2012, com o número 703.376 do parecer de aprovação.

\section{RESULTADOS E DISCUSSÃO}

O projeto de extensão "Farmacovigilância na segurança do Paciente", da Universidade Estadual do Sudoeste da Bahia, foi criado com intuito de desenvolver ações de farmacovigilância e segurança do paciente visando à detecção, avaliação e compreensão de eventos adversos, erros de administração, de prescrição e de dispensação ou quaisquer outros possíveis problemas relacionados a medicamentos.

O caminho para construção e operacionalização do projeto de extensão foi iniciado após a aprovação do projeto na PróReitoria de extensão da Universidade Estadual do Sudoeste da Bahia, que disponibilizou duas bolsas de extensão para alunos de graduação em Farmácia.

Posteriormente, iniciaram-se os trabalhos de pesquisa bibliográfica os quais foram encontrados 86 artigos. Os relatos de experiência e ensino foram incluídos no estudo, totalizando 24 artigos para leitura e referência.

Houve treinamento dos alunos envolvidos, desenvolvimento de protocolos de dispensação de medicamentos, desenvolvimento de instrumentos de coletas e divulgação do projeto no hospital com palestras e cartazes. A equipe do projeto a partir de então não conseguiu prosseguir muito bem, pois acreditava que faltava a plena divulgação e treinamento da equipe do hospital envolvendo o tema de segurança do paciente. 
Em virtude dos problemas e dificuldades encontradas na realização do projeto, receio dos profissionais em relatarem os eventos adversos relacionados a medicamentos, falta de uma cultura voltada para a segurança do paciente, dificuldades na finalização dos trabalhos, falta de notificações no Sistema de Notificações em Vigilância Sanitária (NOTIVISA) da Agência Nacional de Vigilância Sanitária (ANVISA), incompletude dos dados nos prontuários dos pacientes para verificação e validação das reações adversas, falta de medicamentos e despreparo da equipe em assumir o serviço de farmacovigilância, foi sugerido o desenvolvimento de um plano operativo do Planejamento Estratégico Situacional como ferramenta para auxílio na resolução de problemas para integração ensino-serviço(11), atividade essa que foi desenvolvida no Curso de Especialização em Gestão da Assistência Farmacêutica do Ministério da Saúde e Universidade Federal de Santa Catarina.

O início das atividades do PES foi marcado pela frase de Carlos Matus "Se planejar é sinônimo de conduzir conscientemente, não existirá então alternativa ao planejamento. Ou planejamos ou somos escravos da circunstância. Negar o planejamento é negar a possibilidade de escolher o futuro, é aceitá-lo seja ele qual for"(15).

O PES é constituído de quatro momentos fundamentais que estão articulados e inter-relacionados, sendo que em cada um há o questionamento básico que deve ser feito pelos planejadores. O primeiro momento é chamado de explicativo e responde às perguntas: "Como?" "Por quê?" "O que é?". O segundo momento é chamado de normativo, no qual se tenta responder a seguinte indagação? "Como deve ser?" O terceiro momento é o estratégico propriamente dito, respondendo à pergunta: "Pode ser?" E o quarto e último momento, chamado tático-operacional, responde ao questionamento: "Como deve ser?"(16).

O momento explicativo foi realizado em uma oficina única com participação de sete dos quatorze atores convidados: estudantes do curso de Farmácia da Universidade Estadual do Sudoeste da Bahia e integrantes do projeto, coordenador da farmácia hospitalar, médico assistente do hospital e um farmacêutico colaborador do projeto. Nesse momento foi realizado o levantamento de diferentes problemas, com base na realidade e vivência de cada ator presente na oficina, conforme Quadro 1, com registro da discussão envolvida nesta oficina.

Quadro 1. Lista de Problemas identificados na oficina do momento explicativo do plano operativo do projeto de extensão em Farmacovigilância em um Hospital Geral na Bahia.

\begin{tabular}{|c|c|}
\hline Número & Problema identificado \\
\hline 01 & Conhecimento insuficiente sobre o projeto \\
\hline 02 & Treinamento insuficiente dos integrantes do projeto e profissionais do hospital \\
\hline 03 & Baixa interação entre os integrantes do projeto e os profissionais do hospital \\
\hline 04 & Baixo compromisso dos integrantes do projeto e dos profissionais do hospital \\
\hline 05 & Falta de um núcleo de farmacovigilância no hospital \\
\hline 06 & Tecnologia da informação insuficiente no hospital \\
\hline 07 & Falta constante de medicamentos no hospital \\
\hline 08 & Falta de profissionais farmacêuticos no hospital \\
\hline 09 & Falta de recursos financeiros \\
\hline 10 & Falta de preenchimento de dados nos prontuários \\
\hline
\end{tabular}

Em seguida foi realizada a priorização de um dos problemas, utilizando a matriz de priorização de problemas do PES que considera como critérios: magnitude, transcendência, vulnerabilidade, urgência e factibilidade. Após a somatória das 
pontuações atribuídas para cada problema, foi priorizado "Baixa interação entre os integrantes do projeto e os profissionais do hospital". O problema priorizado foi trabalhado pela equipe que definiu os descritores, causas e consequências deste, além de uma imagem objetivo seguindo o modelo de diagrama de causa e efeito de Ishikawa(17) (1993), conhecido como diagrama "espinha de peixe" (Figura 1).

Figura 1. Espinha de peixe do momento explicativo do plano operativo do projeto de extensão em Farmacovigilância em um Hospital Geral na Bahia

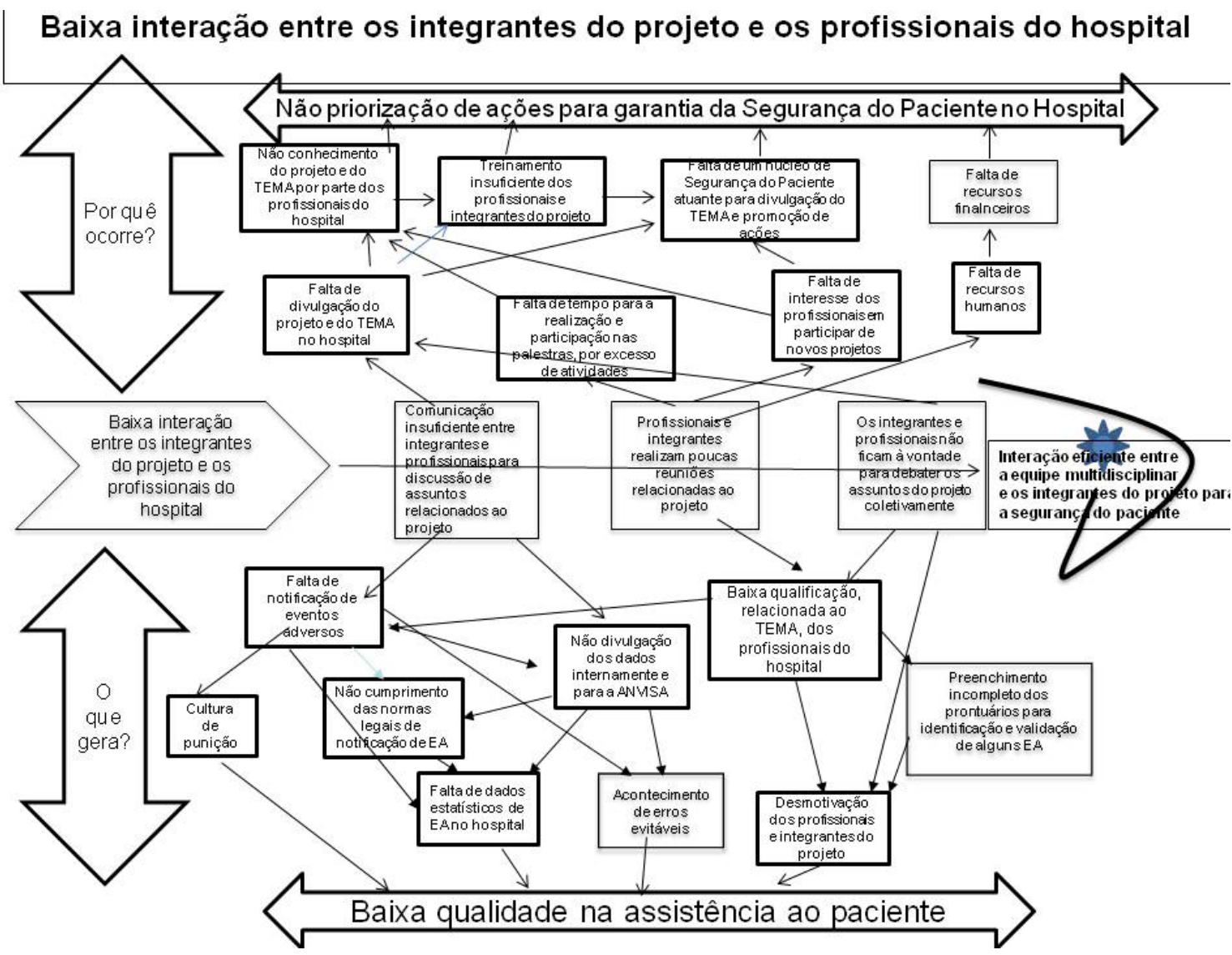

Na oficina do Momento Explicativo estavam representados o setor de farmácia do hospital, a equipe de assistência e o projeto de extensão, foco do plano operativo. Essa foi uma experiência muito positiva para todos os profissionais e alunos que puderam interagir no descobrimento de atividades relacionadas ao serviço que poderiam ser executadas e trabalhadas no ensino, verificando a possibilidade de cooperação com os funcionários do hospital em suas atividades diárias e identificando possíveis "situações-problema" na atividade de extensão proposta, portanto a gestão do trabalho deve integrar todos os trabalhadores, priorizando a liberdade, solidariedade e responsabilização para que todos individualmente se sintam parte da equipe ${ }^{(11)}$. 
A priorização dos problemas deve levar em conta o impacto que sua resolução pode produzir, incluindo tanto seu efeito sobre a percepção dos atores, quanto sobre os demais problemas identificados ${ }^{(18)}$. Uma vez eleitos pelo grupo, os problemas, descritores e nós críticos foram analisados, verificando sua pertinência em relação ao projeto de extensão de Farmacovigilância e sua relevância para os atores locais com a construção da "espinha de peixe".

Esse exercício nos possibilitou uma aproximação maior entre a equipe multiprofissional do hospital e os integrantes do projeto (coordenadora).

A espinha de peixe é o resumo e visualização de toda a discussão levantada na oficina, com descrição do problema priorizado, descritores, causas e consequências rumo à imagem-objetivo (coordenadora).

A descrição dos problemas, contextualizados à realidade analisada, deve traduzir, assim, o diálogo realizado entre os diferentes atores, os quais explicitam suas posições, conscientes de sua coexistência numa realidade conflitiva ${ }^{(19)}$.

Os momentos normativo e estratégico foram realizados em encontros distintos com os diferentes atores que participaram do primeiro momento. No momento normativo foi delineado o plano de intervenções, que se caracteriza por definir operações e ações concretas com o objetivo de solucionar o problema priorizado no momento explicativo (Quadro 2).

Quadro 2. Matriz do momento normativo: objetivos, operações e ações do plano operativo do projeto de extensão em Farmacovigilância em um Hospital Geral na Bahia.

\begin{tabular}{|c|c|c|}
\hline $\begin{array}{l}\text { OBJETIVOS } \\
\text { ESPECÍFICOS }\end{array}$ & OPERAÇÕES & AÇÕES \\
\hline \multirow{2}{*}{$\begin{array}{l}\text { Divulgar o projeto e o tema } \\
\text { "Segurança do paciente" } \\
\text { para cumprimento das } \\
\text { normas legais nacionais no } \\
\text { hospital }\end{array}$} & $\begin{array}{c}\text { Criar um projeto de } \\
\text { divulgação do projeto e do } \\
\text { tema }\end{array}$ & $\begin{array}{l}\text { Avaliar os recursos financeiros disponíveis } \\
\text { Criar um plano para captação de recursos } \\
\text { Responsabilizar um funcionário para divulgação }\end{array}$ \\
\hline & $\begin{array}{l}\text { Promover a divulgação do } \\
\text { projeto e do tema }\end{array}$ & $\begin{array}{l}\text { Criar um grupo virtual para a divulgação de ações } \\
\text { Criar um boletim interno para divulgação das ações }\end{array}$ \\
\hline \multirow{2}{*}{$\begin{array}{l}\text { Capacitar os profissionais } \\
\text { e integrantes do projeto } \\
\text { para realização de ações } \\
\text { assistenciais seguras }\end{array}$} & $\begin{array}{l}\text { Elaborar um projeto de } \\
\text { educação continuada e } \\
\text { capacitação }\end{array}$ & $\begin{array}{l}\text { Identificar fontes de recursos financeiros } \\
\text { Elaborar um projeto para captação de recursos } \\
\text { Definir cronograma e escala para a participação }\end{array}$ \\
\hline & $\begin{array}{l}\text { Capacitar os envolvidos no } \\
\text { projeto }\end{array}$ & $\begin{array}{l}\text { Realizar oficinas de treinamento e capacitação } \\
\text { Oferecer benefícios (certificados) aos participantes } \\
\text { Criar grupo de estudo para Segurança do paciente }\end{array}$ \\
\hline \multirow{2}{*}{$\begin{array}{l}\text { Adequar a quantidade } \\
\text { de pessoal aos serviços } \\
\text { assistenciais para um } \\
\text { atendimento seguro }\end{array}$} & $\begin{array}{c}\text { Avaliar necessidade de } \\
\text { recurso humano no Núcleo } \\
\text { de Segurança do Paciente } \\
\text { (NSP) }\end{array}$ & \multirow{2}{*}{$\begin{array}{l}\text { Verificar se os profissionais estão sendo qualificados com relação } \\
\qquad \text { ao tema } \\
\text { Avaliar se existem dados estatísticos de erros e Eventos Adversos } \\
\text { relacionados a medicamentos } \\
\text { Verificar se os dados estão informados na ANVISA } \\
\text { Delegar responsáveis para a formação do núcleo } \\
\text { Capacitar os responsáveis integrantes do núcleo } \\
\text { Separar um espaço físico estruturado para o núcleo }\end{array}$} \\
\hline & $\begin{array}{l}\text { Definir o Núcleo de } \\
\text { Segurança do Paciente }\end{array}$ & \\
\hline
\end{tabular}


No momento estratégico foi realizada uma avaliação da possibilidade de execução do plano considerando as dimensões política, econômica, competências técnica e administrativa. A matriz do momento estratégico com a análise da viabilidade (decidir, executar e manter) e a análise da factibilidade (recursos existentes e recursos necessários) foram desenvolvidas para todas as ações propostas no momento normativo, além da avaliação dos déficits e atividade estratégica. Nesta última, foram propostas a sensibilização do gestor da Direção geral do hospital, a criação de alianças, a apresentação da obrigatoriedade do tema e a importância dessas ações na assistência para que o projeto de divulgações sobre o tema fosse desenvolvido mais conscientemente. Ressalta-se que a sensibilização do gestor e a criação de alianças já foram implementadas.

No Momento Estratégico enfatiza-se a importância de analisar recursos econômicos, administrativos e políticos (viabilidade e factibilidade) necessários e/ou disponíveis, por meio da matriz do momento estratégico. O momento estratégico é um diferencial do PES, pois traz à reflexão as situações descritas no momento normativo (o que deve ser), mas que por algum motivo não estão em condições de serem executadas, representadas como déficit. Portanto, deve-se construir mecanismos para viabilizar a execução dessas ações e operações essenciais para o plano.

O desenvolvimento do momento normativo nos faz repensar sobre todos os serviços desenvolvidos no projeto e no hospital, com proposição de ações que fazem parte do dia a dia dos profissionais e alunos que pareciam estarem adormecidas (coordenadora).

A etapa do Momento Normativo propõe a definição de objetivos e resultados a alcançar, bem como a previsão de estratégias e ações necessárias para seu alcance, denominada por Teixeira(18) $(1995)$ de desenho da situação-objetivo. Nesse momento, os participantes puderam fazer uma revisão da espinha de peixe traçando um desenho da situação, elencando objetivos e planos de ação.

Na finalização do Plano, momento tático-operacional, foi necessário o estabelecimento de indicadores de monitoramento e avaliação para o acompanhamento de sua execução. Esses indicadores foram definidos por meio do protocolo de indicadores e a matriz final do plano operativo (Quadro 3).

Quadro 3. Matriz final do plano operativo do projeto de extensão em Farmacovigilância em um Hospital Geral na Bahia.

\begin{tabular}{|c|c|c|c|c|c|c|}
\hline \multirow{2}{*}{$\begin{array}{l}\text { Objetivos } \\
\text { Específicos }\end{array}$} & \multirow{2}{*}{ Operações } & \multirow{2}{*}{ Ações } & \multicolumn{2}{|c|}{ Responsabilidade e Centralidade } & \multirow{2}{*}{$\begin{array}{l}\text { Prazo } \\
\text { para as } \\
\text { ações }\end{array}$} & \multirow{2}{*}{$\begin{array}{l}\text { Indicador de } \\
\text { avaliação } \\
\text { (operação) }\end{array}$} \\
\hline & & & Ator principal & Parceiros & & \\
\hline \multirow{2}{*}{$\begin{array}{l}\text { Divulgar o projeto e } \\
\text { o tema Segurança } \\
\text { do paciente para } \\
\text { cumprimento das } \\
\text { normas legais }\end{array}$} & $\begin{array}{l}\text { Criar um projeto } \\
\text { de divulgação do } \\
\text { projeto e do tema }\end{array}$ & $\begin{array}{l}\text { Recursos financeiros } \\
\text { disponíveis } \\
\text { Plano de captação de recurso } \\
\text { Funcionário para divulgação }\end{array}$ & \multirow{2}{*}{$\begin{array}{c}\text { Projeto de } \\
\text { extensão e } \\
\text { profissionais } \\
\text { do núcleo de } \\
\text { segurança do } \\
\text { paciente }\end{array}$} & $\begin{array}{l}\text { Profissionais do } \\
\text { setor administrativo } \\
\text { e financeiro }\end{array}$ & Imediato & $\begin{array}{l}\text { Projeto criado } \\
\text { e consolidado }\end{array}$ \\
\hline & $\begin{array}{c}\text { Promover a } \\
\text { divulgação do } \\
\text { projeto e do tema }\end{array}$ & $\begin{array}{c}\text { Grupo virtual de divulgação } \\
\text { Boletim interno para } \\
\text { divulgação }\end{array}$ & & $\begin{array}{l}\text { Profissionais } \\
\text { e técnicos da } \\
\text { assistência e em } \\
\text { informática }\end{array}$ & $\begin{array}{l}\text { Imediato } \\
3 \text { meses }\end{array}$ & $\begin{array}{c}\% \text { de } \\
\text { divulgações } \\
\text { realizadas }\end{array}$ \\
\hline
\end{tabular}




\begin{tabular}{|c|c|c|c|c|c|c|}
\hline \multirow{3}{*}{$\begin{array}{c}\text { Capacitar os } \\
\text { profissionais e } \\
\text { integrantes do } \\
\text { projeto para } \\
\text { realização de ações } \\
\text { assistências seguras }\end{array}$} & $\begin{array}{l}\text { Elaborar um } \\
\text { projeto de } \\
\text { educação } \\
\text { continuada e } \\
\text { capacitação }\end{array}$ & $\begin{array}{l}\text { Fontes de recursos financeiros } \\
\text { Projeto: captação de recursos } \\
\text { Cronograma de participação }\end{array}$ & \multirow{3}{*}{$\begin{array}{c}\text { Projeto de } \\
\text { extensão e } \\
\text { profissionais } \\
\text { do núcleo de } \\
\text { segurança do } \\
\text { paciente, gestor } \\
\text { do hospital }\end{array}$} & $\begin{array}{c}\text { Profissionais do } \\
\text { setor administrativo } \\
\text { e financeiro }\end{array}$ & $\begin{array}{l}\text { Imediato } \\
1 \text { mês }\end{array}$ & $\begin{array}{l}\text { Projeto criado } \\
\text { e consolidado }\end{array}$ \\
\hline & \multirow{2}{*}{$\begin{array}{l}\text { Capacitar os } \\
\text { envolvidos no } \\
\text { projeto }\end{array}$} & $\begin{array}{l}\text { Treinamento e capacitação } \\
\text { Certificados aos participantes }\end{array}$ & & $\begin{array}{l}\text { Profissionais } \\
\text { convidados }\end{array}$ & \multirow[b]{2}{*}{3 meses } & \multirow{2}{*}{$\begin{array}{l}\text { \%de } \\
\text { capacitações } \\
\text { realizadas }\end{array}$} \\
\hline & & $\begin{array}{l}\text { Grupo de estudo para } \\
\text { segurança do paciente }\end{array}$ & & $\begin{array}{c}\text { setor administrativo } \\
\text { e financeiro } \\
\text { Profissionais } \\
\text { convidados }\end{array}$ & & \\
\hline \multirow{2}{*}{$\begin{array}{c}\text { Adequar a } \\
\text { quantidade de } \\
\text { pessoal aos serviços } \\
\text { assistenciais para um } \\
\text { atendimento seguro }\end{array}$} & $\begin{array}{c}\text { Avaliar } \\
\text { necessidade de } \\
\text { recurso humano } \\
\text { no núcleo de } \\
\text { segurança do } \\
\text { paciente }\end{array}$ & \multirow{2}{*}{$\begin{array}{l}\text { Profissionais estão sendo } \\
\text { qualificados } \\
\text { Existem dados estatísticos de } \\
\text { eventos adversos } \\
\text { Dados informados na ANVISA } \\
\text { Responsáveis pela formação } \\
\text { do núcleo } \\
\text { Capacitar os integrantes do } \\
\text { NSP }\end{array}$} & $\begin{array}{c}\text { Profissionais do } \\
\text { NSP }\end{array}$ & $\begin{array}{l}\text { Setor de recursos } \\
\text { humanos e setor } \\
\text { financeiro }\end{array}$ & \multirow[t]{2}{*}{3 meses } & $\begin{array}{l}\text { Necessidade } \\
\text { de } \\
\text { funcionários } \\
\text { identificada }\end{array}$ \\
\hline & $\begin{array}{c}\text { Definir o núcleo } \\
\text { de segurança do } \\
\text { paciente }\end{array}$ & & $\begin{array}{c}\text { Gestor do } \\
\text { hospital } \\
\text { Profissionais do } \\
\text { NSP }\end{array}$ & $\begin{array}{l}\text { Profissionais } \\
\text { convidados }\end{array}$ & & $\begin{array}{l}\text { Organograma } \\
\text { criado e } \\
\text { implementado }\end{array}$ \\
\hline
\end{tabular}

O momento tático-operacional prevê a programação da implementação das propostas, incluindo cronograma, recursos, atores responsáveis e participantes na execução. No entanto, ele não foi executado durante o plano operativo do PES. Nesse instante foi possível analisar sobre a competência dos diferentes atores para o enfrentamento e a resolução dos problemas, revelando a dependência do projeto de extensão Farmacovigilância na segurança do paciente com o gestor geral do hospital e os coordenadores do serviço no preparo de estratégias para obtenção de governabilidade e planejamento ${ }^{(20)}$. Além disso, nessa etapa os participantes confrontaram-se com as dificuldades inerentes ao processo de definição de indicadores de avaliação frente aos objetivos propostos.

A teoria às vezes se distancia da prática, por isso, conhecer como cada um trabalha, as suas dificuldades, limitações, prioridades, é muito importante e torna o planejamento estratégico situacional uma ferramenta de grande utilidade para avaliação de problemas, proposição de objetivos, operações, ações e metodologias de avaliação das ações (indicadores). Assim como descrito por outros atores ${ }^{(11,19,20)}$, o exercício do PES na integração ensino-serviço em hospitais e na estratégia da saúde da família permitiu aos participantes o desenvolvimento de competências de comunicação, liderança, tomada de decisões, administração e gerenciamento, além de gerar conhecimentos e promover uma aproximação entre atores do ensino-extensão e do serviço de assistência hospitalar. Além das ações propostas no plano operativo do Planejamento Estratégico Situacional, outras também puderam ser avaliadas, discutidas e implementadas a partir da realização do exercício do PES.

Essas propostas de intervenções já estavam em andamento, com apoio do gestor do hospital, da Comissão de Controle de Infecção Hospitalar $(\mathrm{CClH})$, coordenação de Enfermagem, coordenação de Farmácia e profissionais do hospital. Diferentes atividades já foram realizadas para atender ao Programa Nacional de Segurança do Paciente como: criação de protocolos 
internos, padronização da lista de medicamentos e avaliação de prescrições de antimicrobianos para promoção do uso racional destes. Outra intervenção implantada e em andamento é o projeto Farmalegria, que foi criado após o contato dos alunos do projeto de extensão Farmacovigilância na segurança do paciente com o ambiente de cuidado da saúde no hospital, especialmente após a percepção da necessidade de humanizar o cuidado ao paciente. O Farmalegria conta com o apoio de diferentes profissionais na instituição de saúde, bem como de membros da comunidade, motivados pelo lema: "Para alegrar um coração faça uma boa ação".

Além do objetivo do plano operativo de auxiliar na implementação do projeto, outros objetivos ligados a esse também puderam ser alcançados, pois aproximaram o profissional farmacêutico do paciente, mudando a postura comumente empregada atualmente, passando o paciente como foco de seu trabalho. Nesse sentido, implementou-se uma parceria entre médicos, farmacêuticos e equipe de enfermagem para implantar ações do uso racional de antimicrobianos, avaliação e intervenções que têm mudado o desfecho para muitos pacientes. Então, o grande desafio, do ponto de vista gerencial, é sair dos meios e pensar na finalidade. E no caso da saúde torna-se imperativo, porque não estamos nos referindo a um resultado qualquer, mas sim à saúde da população. Assim, o farmacêutico deve estar integrado ao processo de cuidado do usuário e à equipe de saúde, tendo como foco a garantia do alcance de resultados terapêuticos positivos ${ }^{(21)}$.

A realização do PES abriu portas aos integrantes do projeto e profissionais do hospital para a execução de outras atividades voltadas para a segurança e humanização do paciente, e permitiu que gestor, coordenadores, profissionais de enfermagem e os próprios farmacêuticos do hospital tivessem uma percepção diferente da contribuição do profissional farmacêutico ao cuidar do paciente.

Assim, quando começamos foi estranho e ao mesmo tempo encantador, estranho porque não havia estudantes de farmácias e farmacêuticos diariamente na rotina das clinicas, e encantador por estar no ambiente diferente da universidade e pelo aprendizado que é muito valioso, pois é uma experiência para a vida toda. É difícil descrever o que sinto quando vou ao hospital (aluna).

Os benefícios da realização do plano operativo do momento estratégico situacional relacionam-se ao conhecimento gerado no sentido de aperfeiçoar a assistência ao paciente com adoção de medidas educativas e de processo na prática diária dos profissionais e alunos, para maior segurança e melhor assistência aos pacientes. Além disso, todas as intervenções propostas, após o desenvolvimento do plano operativo, que estão em andamento, buscam fornecer um melhor atendimento dos beneficiários e melhores condições para os profissionais do hospital. E finalmente a experiência proporcionada aos alunos, futuros profissionais de saúde, foi muito importante gerando um enorme conhecimento técnico e pessoal.

\section{CONSIDERAÇÕES FINAIS}

As intervenções definidas por meio da proposta do plano operativo do Planejamento Estratégico Situacional foram válidas em diferentes intensidades, sendo que as vivências subsidiaram o crescimento dos participantes ao possibilitar as intervenções de maneira estratégica atuando em problemas do cotidiano do processo de trabalho dos profissionais no hospital. O enfrentamento de problemas, a partir de um olhar abrangente, considerando a participação de todos os atores envolvidos, foi um desafio para todos.

Na teoria, a necessidade de avaliar deve estar integrada ao próprio processo de trabalho. Não podemos apenas apagar incêndio a todo o momento, por isso, planejar é preciso, principalmente quando trabalhamos o cuidado em saúde.

Frente ao exposto, verificamos que a ferramenta do Planejamento Estratégico Situacional foi adequada para o planejamento de serviços em saúde e resolução de problemas, assim como uma estratégia de trabalho adequada na graduação de cursos da área de saúde. 


\section{REFERÊNCIAS}

1. Velo GP, Minuz, P. Medication errors: prescribing faults and prescription errors. Br. J. Clin. Pharmacol. 2009; 67(6):624-8.

2. Brasil. Agência Nacional de Vigilância Sanitária. RDC no 36 de 25 de julho de 2013c. Institui ações para a segurança do paciente em serviços de saúde e dá outras providências.

3. Neri EDR, et al. Erros de prescrição de medicamentos em um hospital brasileiro. Rev Assoc Med Bras. 2012, 57(3):306-314.

4. Needleman J, Buerhaus P, Pankratz S, Leibson CL, Stevens SR, Harris M. Nursestaffing and in patient hospital mortality. New England Journal of Medicine. England. 2011; (364):1037-45.

5. Rosa, M.B. et al. Legibilidade de prescrições médicas com medicamentos potencialmente perigosos em um hospital em Belo Horizonte, MG, 2001. Rev.Soc.Bras. Farm. Hospitalar. 2003; (2):22-28.

6. Wilson RM, Michel P, Olsen S, Gibberd RW, Vincent C, El-Assady R. et al. A segurança dos pacientes nos países em desenvolvimento: estimativa retrospectiva de escala e natureza dos danos aos pacientes no hospital. BMJ. 2012, 344: E832.

7. Mendes W, Martins M, Rozenfeld S, Travassos C. The assessment of adverse events in hospitals in Brazil.International Journal for Quality in Health Care. 2009, 21(4):279-84.

8. ASPDENP, et al. (ed.). Committee on Identifying and Preventing Medication Errors. Preventing medication errors Institute of Medicine of the National Academies. Washington, D.C.: The National Academies Press, 2007, 544 p. (Quality Chasm Series).

9. Brasil. Ministério da Saúde. Portaria n 529 de 01 de abril de 2013b. Institui o Programa Nacional de Segurança do Paciente. Publicada no DOU em 02 de abril de 2013.

10. Brasil. Ministério da Saúde. Portaria № 3.410, de 30 de dezembro de 2013. Estabelece as diretrizes para a contratualização de hospitais no âmbito do Sistema Único de Saúde (SUS) em consonância com a Política Nacional de Atenção Hospitalar (PNHOSP).

11. Vendruscolo C, Kleba ME, Krauzer IM, Hillesheim A. Planejamento situacional na Estratégia Saúde da Família: atividade de integração ensino-serviço na enfermagem. Rev. Gaúcha Enferm. 2010, 31(1):183-6.

12. Matus C. Política, planejamento \& governo. Brasília: Ipea, 1996.

13. Santana R.S; Lobo I.M.F; Penaforte TR, Leite SN, Silva WB. A institucionalização da seleção de medicamentos em hospitais públicos por meio do planejamento estratégico situacional. Rev. Adm. Pública — Rio de Janeiro. 2014 nov./dez, 48(6):1587-1603.

14. Contradiopoulos AP A avaliação na área da saúde: conceitos e métodos. In: Hartz Z MA. (Org.). Avaliação em saúde: dos modelos conceituais à prática na análise da implantação de programas. Rio de Janeiro: Editora Fiocruz, 1997, 29-47.

15. Leite SN, Guimarães MCL. Gestão da assistência farmacêutica. In: Brasil. Gestão da assistência farmacêutica [Recurso eletrônico] / Universidade Federal de Santa Catarina, Universidade Aberta do SUS. Florianópolis: UFSC, 2013.

16. Gentilini JA. Atores, cenários e planos: o planejamento estratégico situacional e a educação. Cadernos de pesquisa. 2014; 44(153):580-601.

17. Ishikawa K. Controle de qualidade total: à maneira japonesa. Rio de Janeiro: Campos, 1993.

18. Teixeira CF. Planejamento e programação situacional em distritos sanitários: metodologia e organização. In: Mendes EV, organizador. Distrito sanitário: o processo social de mudança das práticas sanitárias do Sistema Único de Saúde. São Paulo/ Rio de Janeiro: Hucitec/Abrasco; 1995, 237-265. 
19. Kleba ME, Krauser IM, Vendruscolo C. O planejamento estratégico situacional no ensino da gestão em saúde da família. Texto Contexto Enferm, Florianópolis, 2011 jan.-mar.; 20 (1):184-93.

20. Melleiro MM, Tronchin DMR, Ciampone MHT. O planejamento estratégico situacional no ensino do gerenciamento em enfermagem. Acta Paul Enferm.2005;18(2):165-71

21. Rabelo ML, Borella MLL. Papel do farmacêutico no seguimento farmacoterapêutico para o controle da dor de origem oncológica. Rev Dor. São Paulo. 2013 jan.-mar.;14 (1):58-60.

Submetido em: 25/03/2016

Aceito em: 07/10/2016

Publicado em: 31/12/2017 